\title{
Microemulsion based gel of sulconazole nitrate for topical application
}

\section{Sumedha P Payyal, Narayana Charyulu R*, Sandeep DS, Ravi GS, Anoop Narayanan V}

Department of Pharmaceutics, NGSM Institute of Pharmaceutical sciences, Nitte (Deemed to be University), Paneer, Deralakatte, Mangalore-575018, Karnataka, India.

\section{${ }^{*}$ Corresponding author:}

Narayana Charyulu R,

Professor and Head

Department of Pharmaceutics,

NGSM institute of Pharmaceutical Sciences,

Paneer, Deralakatte, Manglore-575018

Karanataka, India

Mobile no. +919448164750

E-mail: narayana@nitte.edu.in 


\section{ABSTRACT}

Objectives:Sulconazole is a broad spectrum anti-fungal agent of imidazole class used against dermatophytes and other fungi to treat skin infections. The aim of present work was to formulate and evaluate microemulsion based topical sulconazole gel.

Materials and Methods:Microemulsion formulation of sulconazole nitrate was prepared by using oil, surfactant, cosurfactantand water at different ratio. This was then subjected to clarity, particle size analysis, centrifugation test, dilution test, freeze thawing

Results:Zeta - potential of formulation F1 was found to be -41.3 and stable pH of the microemulsion formulation was found within the range of $\mathrm{pH}$ of skin. The formulation $\mathrm{F} 1$ showed higher amount percentage drug as compared with other formulations. The viscosity showed that the formulation F1 was optimum. The freezing and thawing results showed there was no phase separation and the formulation was found to be stable.In vitrodrug release showed that the drug release from the microemulsion formulation $\mathrm{F} 1$ was found to be higher when compared to other formulations. It revealed F1 formulation with highest drug content of $95.88 \pm 0.3 \%$ and $\%$ cumulative drug release was found to be $88.75 \%$ release in 8 h. In vivo skin irritation study on rats confirmed that formulation was non toxic and non irritant.

Conclusion:The study of present work confirmed the safety of the formulated sulconazole loaded microemulsion gel for topical application.

Keywords: Microemulsion, Sulconazole Nitrate, In Vitro Release, Fungal Infection.

\section{INTRODUCTION}


Novel drug delivery systems are meant to attain and maintain sufficient therapeutic levels of drug at the target site. In order to overcome the problems connected with traditional modes of drug administration topical drug delivery system was started. For local dermatological disorders topical drug delivery is the effective way for drug administration. Drug delivery through skin has fame because it bypasses the first pass effects, gastrointestinal irritation and metabolic degradation associated with oral administration. The topical route of administration is also utilized to produce systemic drug effects in some instances. Major attention is required to new topical formulations that ensure adequate localization of drug within the skin to enhance local effect or to increase the penetration. Drugs applied topically, mainly for local action, include antifungal, anti-inflammatory, antiseptic agents as well as skin emollients for protective effects while this route can also be used for systemic drug delivery. ${ }^{1}$

Microemulsions are thermodynamically stable multicomponent fluids consisting of oil, water and surfactant. The droplets of microemulsions are in the range of $10-100 \mathrm{~nm}$ in diameter. Since the dispersed particles having diameter less than one-fourth of the wavelength of visible light, they do not refract light and that's the reason microemulsions are transparent to the eyes. ${ }^{2,3}$

Microemulsion when applied to the skin is expected to penetrate the stratum corneum and to exist intact to the horny layer, resulting in alteration of both the lipid and the polar pathways. The lipophilic domain of the microemulsion interacts with stratum corneum in the different way. The drug which gets dissolved in the lipid domain of the microemulsion can directly partition into lipid of stratum corneum. ${ }^{4}$

Sulconazole is a broad spectrum anti-fungal agent of imidazole class used against dermatophytes and other fungi to treat skin infections such as ring worm and athlete's foot. It is lipophilic in nature and can be effortlessly formulated for topical delivery by using microemulsion as topical vehicle for antifungal effect with several advantages such as ease of preparation, thermodynamic stability, transparent and elegant appearance, increased drug loading, enhanced penetration to the biological membrane, increased bioavailability compared to conventional dosage form like cream etc. Hence in the present study we developed sulconazole loaded microemulsion gel for topical 
delivery and investigated for physicochemical characterization, drug content, in vitro drug release and kinetic studies and in vivo skin irritation study. ${ }^{5}$

\section{MATERIALS AND METHODS}

\section{Materials}

Sulconazole nitrate was received as a gift sample from Ranbaxy Laboratory, New Delhi, India. Olive oil, Tween 20 and PEG 400 were purchased from HiMedia, Mumbai, India. Propylene glycol and Carbopol 934 were procured from Lobachemie Pvt. Ltd, Mumbai, India. Triethanolaminewas purchased from Rankem Fine Chemicals Ltd., New Delhi, India. All the chemicals and reagents were used of analytical grade.

\section{Methods}

\section{Fourier transform infrared spectroscopy (FTIR)}

FTIR spectroscopy was carried out to check the compatibility between the drug and polymer. Using IR spectrophotometer (Spectrum one model, Alpha-Bruker, Germany) IR spectrum of sulconazole nitrate, carbopol 934 and physical mixture of sulconazole nitrate and carbopol was obtained and compared to check the interactions.

\section{Preperation of microemulsion}

Sulconazolenitrate containing o/w microemulsion was formulated by water titration method which includes following steps. First, surfactant and co-surfactant were added in fixed ratios to oil foliowed by sulconazole nitrate. Then bathsonication was performed for $5 \mathrm{~min}$ followed by heating. Later required quantity of water was added dropwise with constant stirring until formation of a clear and transparent liquid. A series of microemulsion formulations were prepared using tween 20 and PEG 400 as the

surfactant and co-surfactant and olive oil as the oil. In all these formulations, amount of sulconazole nitrate was kept constant $(50 \mathrm{mg})$ and amount of surfactant, co-surfactant, oil and co-solvent were varied(Table 1).

\section{Table 1. Composition of different microemulsion formulations}




\begin{tabular}{|c|c|c|c|c|c|}
\hline $\begin{array}{c}\text { Formulation } \\
\text { Code }\end{array}$ & $\begin{array}{c}\text { Sulconazole } \\
\text { Nitrate }\end{array}$ & Olive oil (\% v/v) & $\begin{array}{c}\text { S:Co S } \\
\text { ratio }\end{array}$ & $\begin{array}{c}\text { Tween20/ } \\
\text { PEG } \\
\mathbf{4 0 0}(\%)\end{array}$ & $\begin{array}{c}\text { Water } \\
\text { (\%) }\end{array}$ \\
\hline F1 & $2 \mathrm{~g}$ & 5.0 & $3: 1$ & 55 & 40.0 \\
\hline F2 & $2 \mathrm{~g}$ & 5.0 & $3: 1$ & 60 & 35.0 \\
\hline F3 & $2 \mathrm{~g}$ & 7.5 & $3: 1$ & 45 & 475 \\
\hline F4 & $2 \mathrm{~g}$ & 7.5 & $3: 1$ & 50 & 42.5 \\
\hline F5 & $2 \mathrm{~g}$ & 10 & $3: 1$ & 55 & 35.0 \\
\hline F6 & $2 \mathrm{~g}$ & 10 & $3: 1$ & 60 & 30.0 \\
\hline
\end{tabular}

\section{Chracterization of sulconazole nitrate microemulsion formulations}

\section{Globule size determination}

One drop of the sample was taken on a microscopic glass slide and coverslip was placed over it and observed at $45 \mathrm{x}$ resolution under microscope (Zeiss, Germany). The particle size was measured using BIOVIS software. ${ }^{6}$

\section{Dilution test}

The formulated microemulsions were diluted with distilled water to confirm type of emulsion and miscibility with the aqueous phase.

\section{Centrifugation test}

This is used to specify the stability of the microemulsion whether it is monophasic or not. In this samples were centrifuged using cold centrifuge (CM8plus- Remi Lab World, Mumbai, India) at $10000 \mathrm{rpm}$ for $30 \mathrm{~min}$ and then are examined for whether the system is monophasic or biphasic. ${ }^{7}$ 


\section{Zeta potential determination}

Malven Zeta Sizer (Malvern Instruments, UK) was used to measure the zeta potential of the globules on the electrophoresis and electrical conductivity of microemulsion. Measurements were performed using small volume zeta cell. ${ }^{7}$

\section{Determination of $\mathrm{pH}$}

$\mathrm{pH}$ of the microemulsion formulations was measured using Digital $\mathrm{pH}$ meter (Systronics 335, Ahmedabad, India).

\section{Determination of viscosity}

The viscosity of the formulated microemulsions was determined by Brookfield viscometer DV-II model using spindle number 92 at 20,30,50 and 60 at $100 \mathrm{rpm}^{7}$

\section{Freeze thawing}

Freeze thawing was employed to evaluate the stability of microemulsion formulations. The microemulsionpreconcentrate of various formulations were subjected to 3 to 4 freeze thaw cycles, which included freezing at $-10^{\circ} \mathrm{C}$ for $24 \mathrm{~h}$ followed by thawing at 40 ${ }^{\circ} \mathrm{C}$ for $24 \mathrm{~h}$. The various formulations were then subjected to centrifugation at $3000 \mathrm{rpm}$ for $5 \mathrm{~min}$. The formulations were then visually observed for phase separation. ${ }^{8}$

\section{Preparation of drug loaded microemulsion gel}

The polymers carbopol 934P and propylene glycol were used to prepare blank gel. Among them carbopol 934P 1\% gel was optimized on its transparency and its consistency for application on skin. Carbopol 934P (1\%) and propylene glycol (5\%) (as humectants) was added to required quantity of water with constant stirring and allowed for hydration for 4-5 h. Microemulsion was to the gel phase and left it overnight. Finally the required quantity of triehanolamine was added to adjust the $\mathrm{pH}^{9}$

\section{Evaluation of microemulsion gel}


The prepared drug containing gel formulations were evaluated for $\mathrm{pH}$ and drug content. For drug content determination, microemulsion gel $(1 \mathrm{~g})$ was weighed \& dissolved in mixture of ethanol:phosphate buffer $\mathrm{pH} 7.4$ (2:3). From this, $2 \mathrm{ml}$ of the solution was diluted to $50 \mathrm{ml}$ with same medium and absorbance was measured at $\lambda_{\max } 227 \mathrm{~nm}$. Blank solution was prepared in the same manner by taking gel without drug. For determination of $\mathrm{pH}, 1 \mathrm{~g}$ of microemulsion gel was dissolved in $100 \mathrm{ml}$ distilled water and stored for $2 \mathrm{~h}$ and $\mathrm{Ph}$ was measured using Digital $\mathrm{pH}$ meter (Systronics 335, Ahmedabad, India). ${ }^{10}$

\section{In vitro drug release and kinetic studies}

In vitro drug release study of drug from formulations was carried out using artificial semi permeable membrane (Cellophane) using Franz diffusion cell. The receptor compartment was consists of $50 \mathrm{ml}$ ethanol:phosphate buffer $\mathrm{pH} 7.4$ (2:3) and temperature was maintained at $37 \pm 2{ }^{\circ} \mathrm{C}$ and stirred using magnetic stirrer. The microemulsion gel ( $10 \mathrm{mg}$ sulconazole nitrate) was placed on artificial semi permeable membrane tied to the one end of open ended glass cylinder that was then dipped into the receptor medium on magnetic stirrer. Samples were taken from the receptor medium for $12 \mathrm{~h}$ at different time intervals and replaced immediately with the buffer. All the samples were analyzed at $227 \mathrm{~nm}$ and the cumulative amount of drug release was calculated. In order to describe the kinetics of the release process of drug in the different formulations, Zero order, First order, Higuchi and Krosmeyer and peppas models were fitted to the dissolution data of selected formulation using linear regression analysis. ${ }^{11}$

\section{In vivo skin irritation study}

The skin irritation test was carried out on the healthy male albino rats $(200-250 \mathrm{~g})$ obtained from NUCARE-Nitte (Deemed to be University) by ethical approval from ethical committee and the document no. is NGSMIPS/IAEC/May-2016/9. Experiments were periormed in accordance with the CPCSEA guidelines. The preparation was applied to the properly shaved skin of rats and secured with adhesive tape, its adverse effects like change in colour, skin morphology, was checked up for $72 \mathrm{~h}$. The total 2 sets of 6 rats 
were used for the study. Among these 6 will be used as placebo and other 6 will be taken as treatment. The animals will be observed for any sign of erythema and edema for the different time intervals. ${ }^{12,13}$

\section{RESULTS}

\section{Fourier transform infrared spectroscopy (FTIR)}

The IR spectrum spectrum of pure drug sulconazole nitrate (Figure 1),carbopol 940P (Figure 2) and physical mixture of sulconazole nitrate with carbopol 940P (Figure 3) was obtained and interpreted for spectral data comparision for computability..(Figure 1).

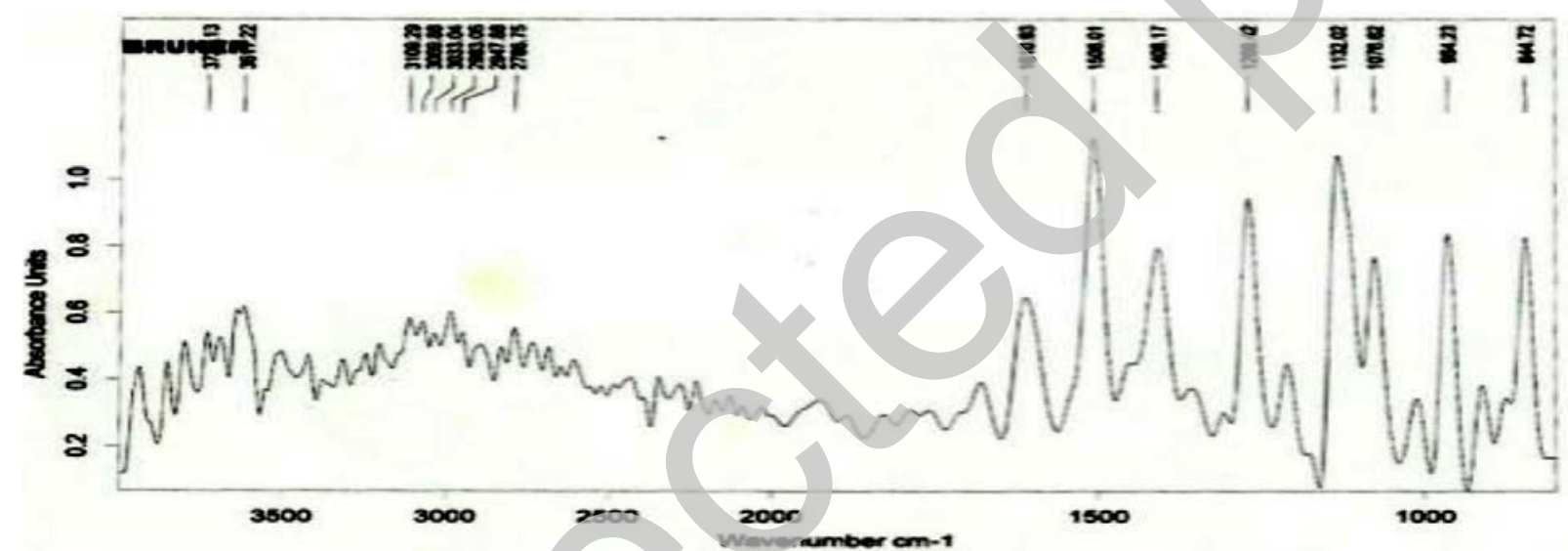

Figure 1. IR spectra of Pure sulconazole nitrate

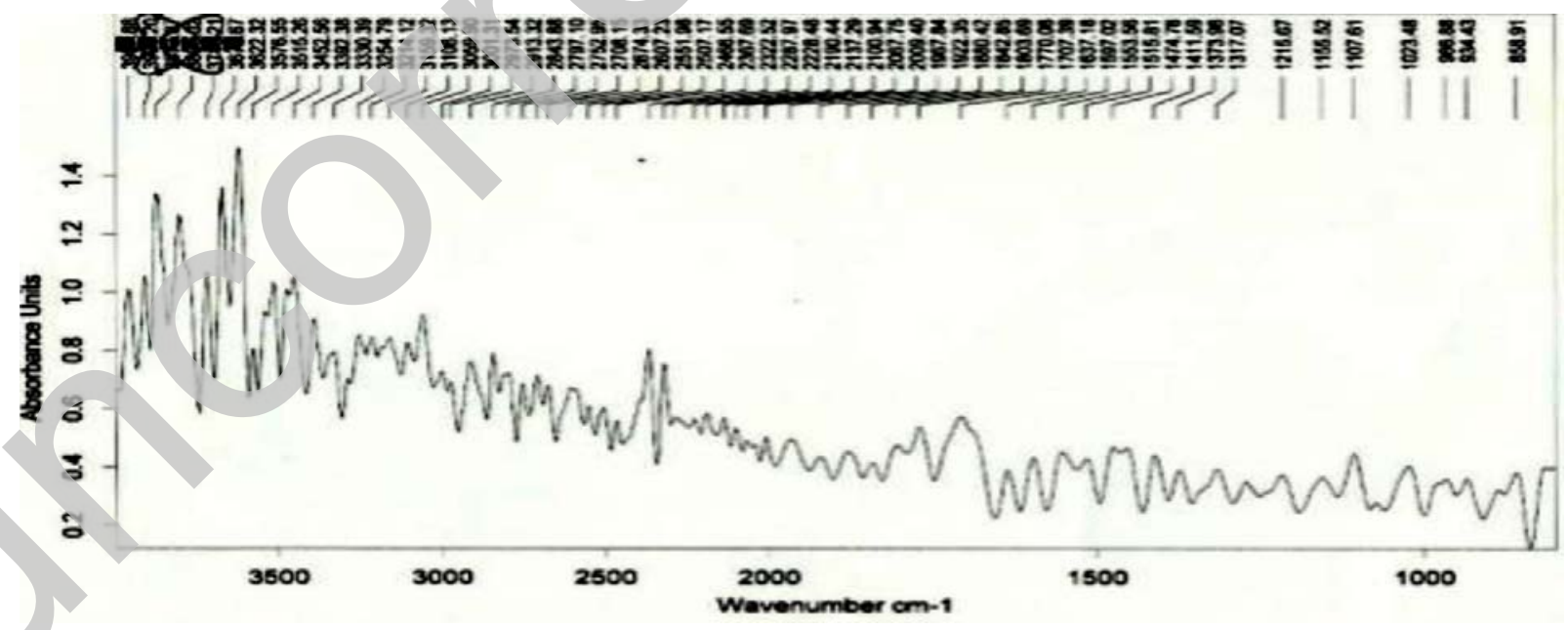

Figure 2. IR spectra of Carbopol 940P 


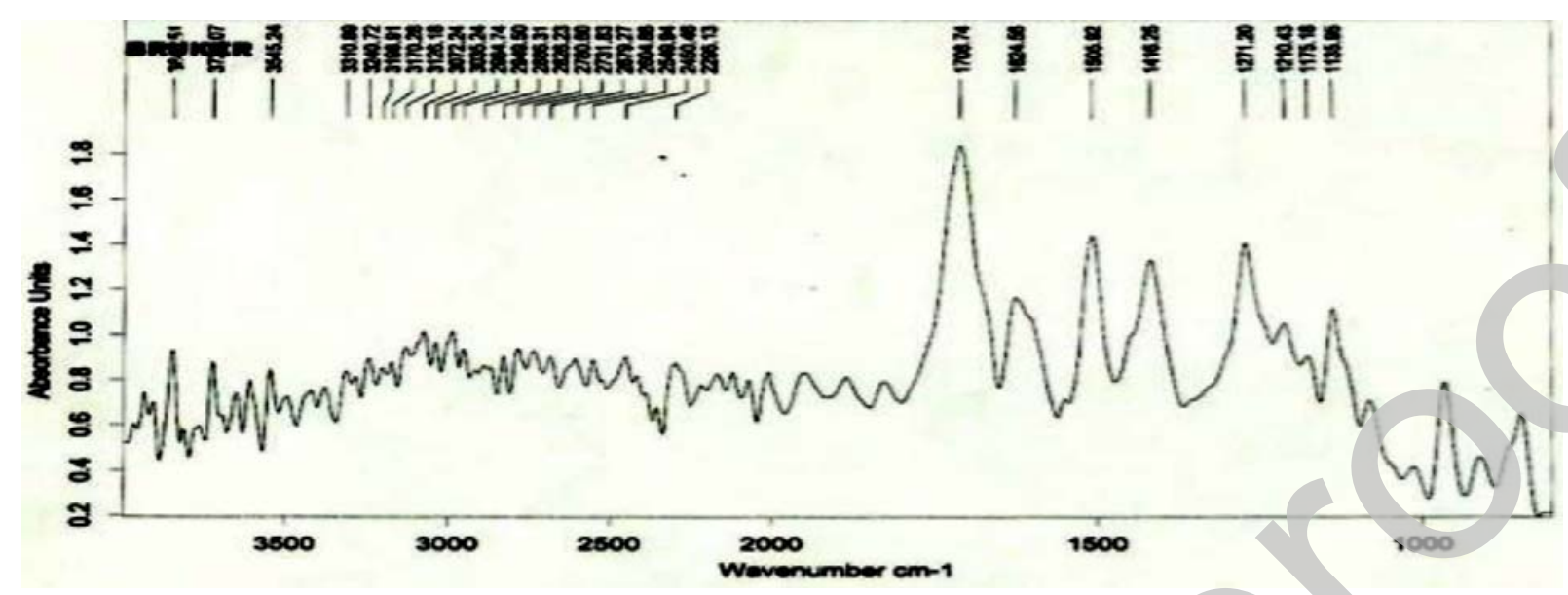

Figure 3. IR spectra of physicalmixture of sulconazole nitrate and Carbopol 934P

\section{Preparation of microemulsion of sulconazole nitrate}

Microemulsion formulations of sulconazole nitrate were preparedby water titration method. According to the definition, microemulsion must be clear and transparent in nature. All the formulations exhibited transparent, clear and without any precipitation which indicated the formation of good microemulsions.

\section{Evaluation of microemulsion of suiconazole nitrate}

\section{Globule size determination}

Microemulsions are thermodynamically stable, transparent systems having particle size $<100 \mathrm{~nm}$. Globule size determination is very important evaluation parameter because therapeutic effectiveness of microemulsion depends on its globule size. The formulated microemulsions showed the globule size between $243.8 \pm 0.02 \mu \mathrm{m}$ to $679.2 \pm 0.01 \mu \mathrm{m}$. Formulation $\mathrm{F} 1$ with the highest proportion of surfactant and co-surfactant at a fixed amount of oil, showed the lowest mean particle diameter whereas formulation F6 with the highest proportion surfactant and fixed amount of oil, showed the highest mean particle diameter.

\section{Dilution test}

Dilution test is based on the fact that emulsion is only miscible with the liquid which forms the continuous phase. 


\section{Centrifugation test}

The data for the formulations for stability for monophasic nature shown in table 2.

Table 2. Evaluation of centrifugation test

\begin{tabular}{|c|c|}
\hline Formulation code & Centrifugation \\
\hline F1 & Monophasic, Stable \\
\hline F2 & Not Stable \\
\hline F3 & Monophasic, Stable \\
\hline F4 & Nonophasic, Stable \\
\hline F5 & Not Stable \\
\hline F6 & Not Stable \\
\hline
\end{tabular}

\section{Freeze thawing test}

The test check the stability and phase separation of formualations if found (Table 3)

Table 3. Results of freeze thawing test

\begin{tabular}{|c|l|}
\hline Formulation Code & Freeze Thawing \\
\hline F1 & Stable and no separation \\
\hline F3 & Stable and no separation \\
\hline F4 & Stable and no separation \\
\hline
\end{tabular}

\section{Determination of zeta potential}

The zeta potential value depends on the type and composition of carrier used in the formulation. The zeta potential of F1 was found to be -33.5 indicates the good stability.(Table 4). 


\section{Determination of $\mathrm{pH}$}

$\mathrm{F} 1, \mathrm{~F} 3$ and $\mathrm{F} 4$ formulations were in the $\mathrm{pH}$ range of $6.32-7.0$ which is well specified range for topical formulations(Table 4).

\section{Drug Content:}

The percentage drug content of F1, F3 and F4 formulations was found to be within a range of $93.05 \%-95.88 \%$. This shows that drug is uniformly distributed throughout the microemulsion. Maximum drug content was obtained for F1 formulation (Table 4).

Table4. Zeta potential, pH and drug content of F1, F3 and F4 formulations

\begin{tabular}{|c|c|c|c|}
\hline Formulation Code & Zeta Potential $(\mathbf{m V})$ & $\mathbf{p H}$ & Drug content (\%) \\
\hline F1 & $-33.5 \pm 2.3$ & $7.0 \pm 0.01$ & $95.88 \pm 0.3 \%$ \\
\hline F3 & $-41.2 \pm 3.4$ & $6.32 \pm 0.03$ & $91.42 \pm 0.2 \%$ \\
\hline F4 & $-38.3 \pm 2.7$ & $6.89 \pm 0.02$ & $93.05 \pm 0.4 \%$ \\
\hline
\end{tabular}

*Mean \pm SD $(n=3)$

\section{Determination of Viscosity}

The viscosity of F1, F2 and F3 microemulsion formulations at 20, 30, 50, 60 and 100 rpm measured using spindle number 92 at room temperature. The viscosity curve was plotted by taking shear rate (rpm) on $x$-axis and viscosity on y-axis (Figure 4) with the data obtained (Table 5).

Table 5. Viscosity of F1, F2 and F3 formulations at different RPM

\begin{tabular}{|c|c|c|c|}
\hline \multirow{2}{*}{ RPM } & \multicolumn{3}{|c|}{ Viscosity(cps) } \\
\cline { 2 - 4 } & F1 & F2 & F3 \\
\hline 20 & $98 \pm 0.01$ & $105 \pm 0.01$ & $89 \pm 0.01$ \\
\hline 30 & $85 \pm 0.07$ & $90 \pm 0.01$ & $81 \pm 0.01$ \\
\hline 50 & $81 \pm 0.02$ & $88 \pm 0.02$ & $76 \pm 0.03$ \\
\hline 60 & $75 \pm 0.03$ & $81 \pm 0.03$ & $70 \pm 0.01$ \\
\hline 100 & $72 \pm 0.02$ & $79 \pm 0.01$ & $68 \pm 0.01$ \\
\hline
\end{tabular}

*Values are mean \pm SD $(n=3)$ 


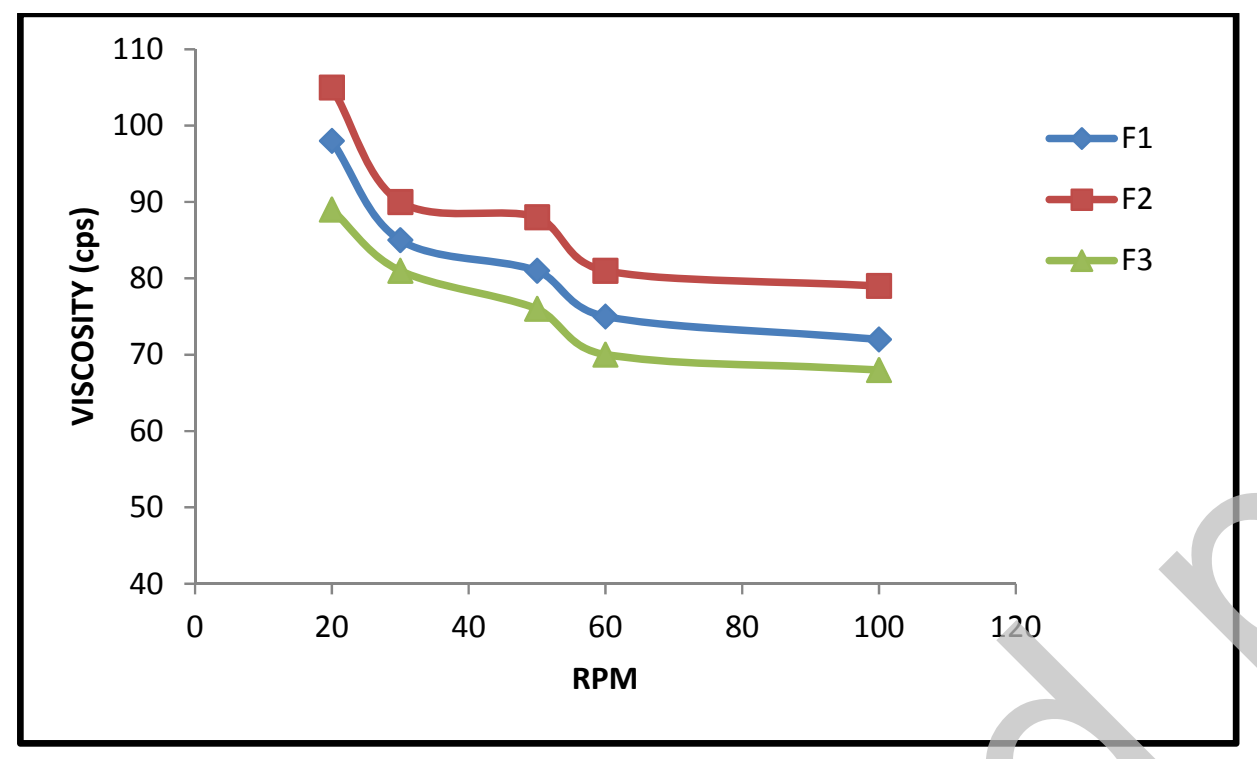

Figure 4. Rheology of Sulconazolemicroemulsions for F1, F3 and F4

\section{Preparation of drug loaded microemulsion gel}

Sulconazole nitrate microemulsion ( $\mathrm{F} 1, \mathrm{~F} 3$ and $\mathrm{F} 4$ ) containing carbapolwas appeared to be transparent, clear and smooth textured. This drug containing gel formulations were further evaluated for $\mathrm{pH}$ and drug content.

\section{Evaluation of microemulision gel}

Drug content values were found to be in the range of $91.5-95.5 \%$ and $\mathrm{pH}$ ranged between 7.2 - 7.8(Table 6 ).

Table 6. Drug content and pH of F1 microemulsion

\begin{tabular}{|c|c|c|c|}
\hline \multirow{2}{*}{ Test } & \multicolumn{3}{|c|}{ Drug loaded microemulsion gel } \\
\cline { 2 - 4 } & F1 & F3 & F4 \\
\hline Drug content & $95.5 \pm 0.34 \%$ & $91.5 \pm 0.26 \%$ & $92.5 \pm 0.36 \%$ \\
\hline pH & $7.21 \pm 0.01$ & $7.72 \pm 0.02$ & $7.81 \pm 0.02$ \\
\hline
\end{tabular}

*Values are mean \pm SD $(n=3)$ 


\section{In vitro drug release and kinetic studies}

Three microemulsion gel formulations (F1 F3 and F4) were selected for the in vitro drug release study. The \% cumulative drug release was $83.32-88.75$ at the end of $8 \mathrm{~h}$ (Figure 5). The mechanism of drug release was calculated by fitting the dissolution data in the different models like Higuchi's and Korsemeyer - Peppas. The best model was selected on the highest regression value (Figure 6).

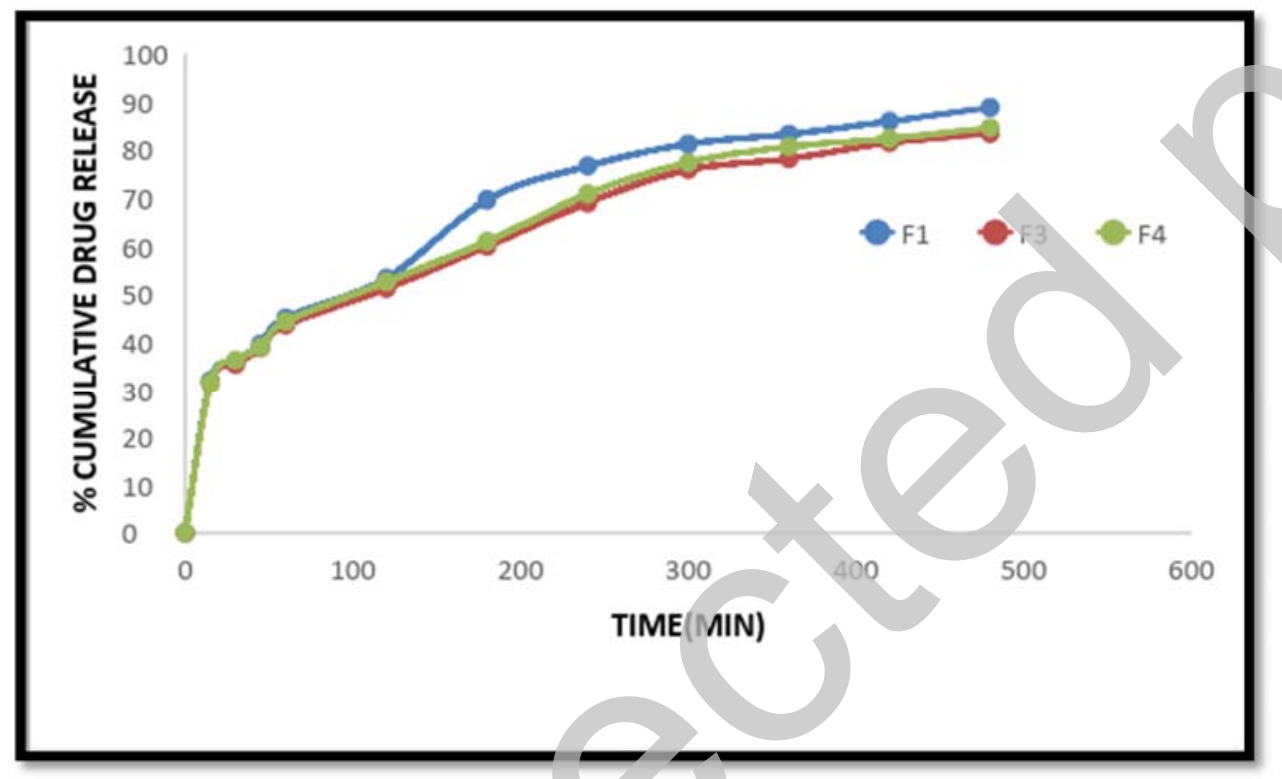

Figure 5. In vitro drug releasedrug release profile of F1, F3 and F4 microemulsion gel formulations 


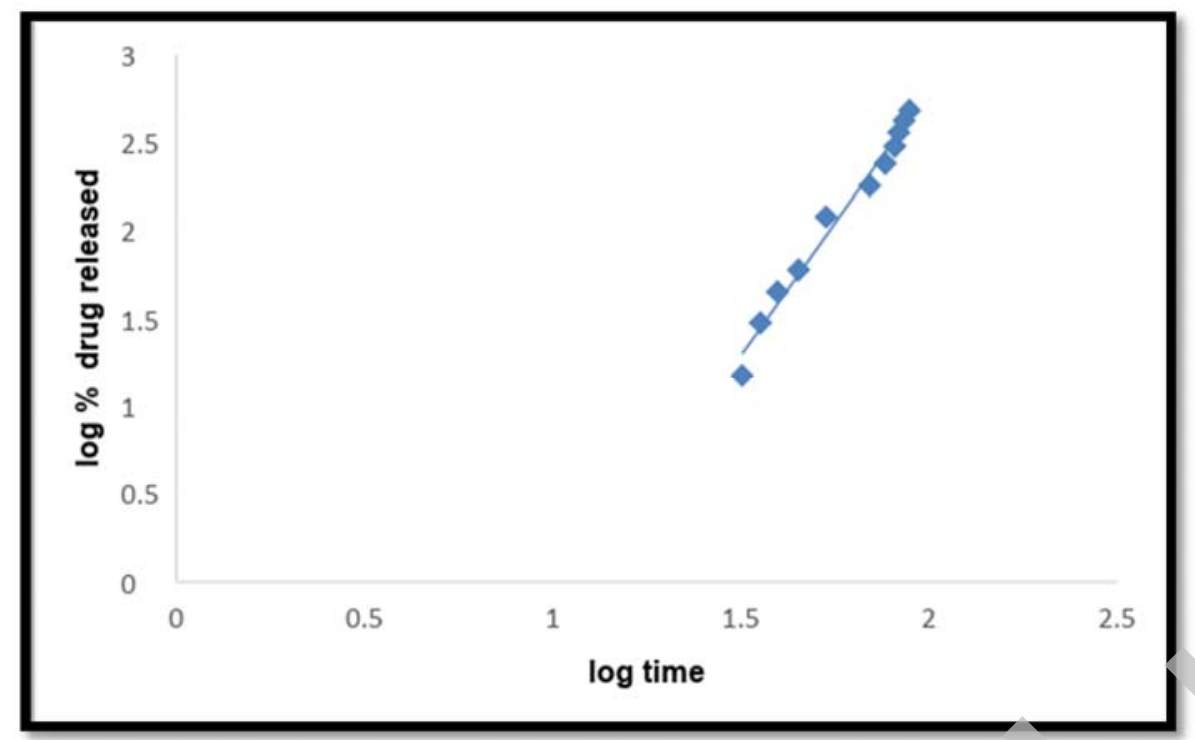

Figure 6. Korsemeyer-Peppas model for F1microemulsion

\section{In vivo skin irritation study}

The skin irritation study was carried out on six rats out of which three were treated with placebo and three with the $\mathrm{F} 1$ formulation observed for $72 \mathrm{~h}$ to check signs of erythema and edema for skin surface (Figure 7)

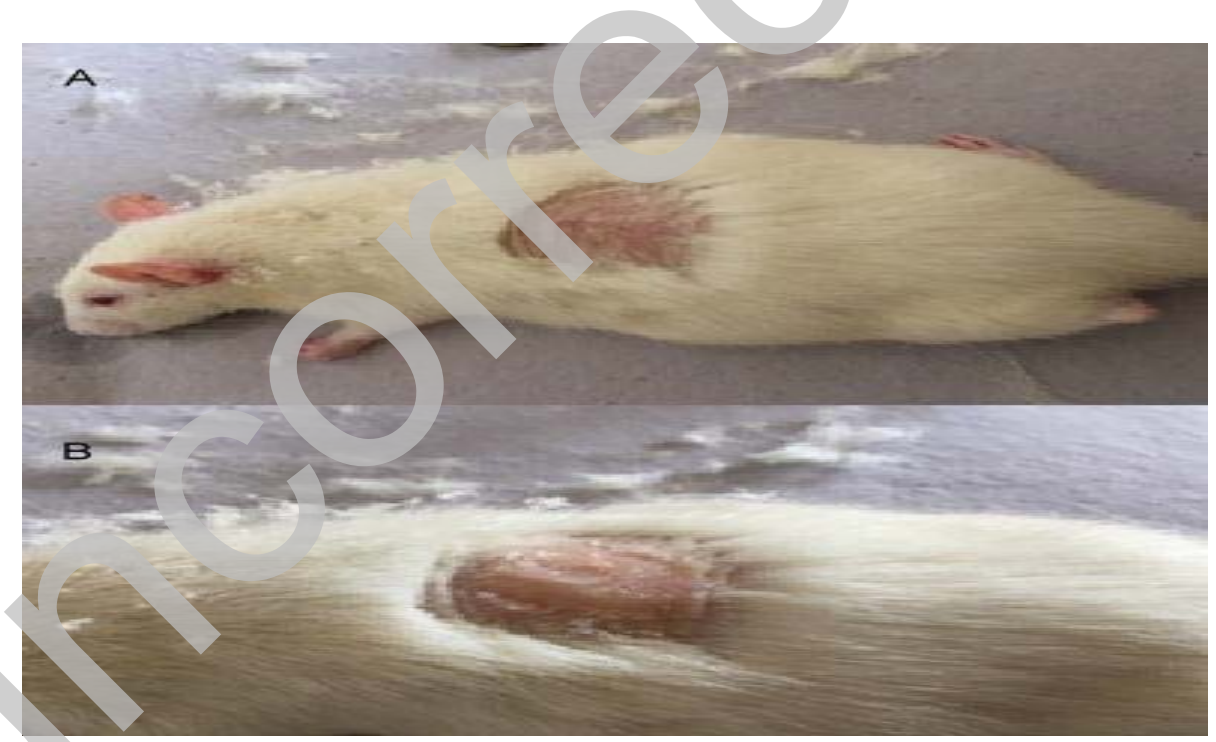

Figure 7. In vivo skin irritation test for F1 microemulsion(A) Before application (B) After application 


\section{DISCUSSION}

From the FTIR study, the peaks appeared in the physical mixture indicated that the drug and the gelling agents are compatible with each other.

From the results of globe size analysis, it was observed that increase in the ratio of the oil phase resulted in the increase in the particle size because of decrease in the surfactant/co-surfactant proportion. An increase in the surfactant/co-surfactant ratio and decrease in oil ratio lead to decrease in mean particle size.

The dilution test showed that after dilution of microemulsion with water, microemulsions were remained clear indicating the good miscibility of microemulsions with water which was used as the continuous phase.

From the centrifugation test, it was observed that F1, F3 and F4 formulations were found to stable and monophasic liquids and further evaluate for $\mathrm{pH}$, drug content and zeta potential.

Freeze thawing results also confirmed that ali three formulations were stable and there was no phase separation.

The zeta potential of formulation $\mathrm{F} 1$ was found to be good and was optimized as best formulation. The $\mathrm{pH}$ of formulated microemulsions was within the specified skin range.

From the viscosity study, ali three formulations showed pseudoplastic non Newtonian flow and viscosity values were decreased as the shear rates increased.

From the drug release data formulation F1 was found to show highest percentage of drug release in comparison to F3 and F4. This may be due to spontaneous formation of microemulsion with small particle size permitted faster rate of drug release. Thus greater permeability of the dissolved sulconazole nitrate from the microemulsion gel formulation which can lead to higher absorption through the skin can be expected.

The formulation F1 followed Korsemeyer - Peppas model since regression coefficient value was higher. 
In vivo skin irritation study showed that there was no sign of erythema and edema after $72 \mathrm{~h}$ of application of the gel and F1 formulation was found to be safe, non-toxic and non-irritant for application on the skin.

\section{CONCLUSION}

The microemulsion based gel of sulconazole nitrate was successfully formulated for topical delivery to treat fungal infections. The formulated gel possessed good physicochemical properties, high drug content and sustained drug release. It is also confirmed that the formulated gel is safer for topical delivery by the in vivo studies. By seeing these results it can be concluded that microemulsion based gel of sulconazole nitrate is promising for topical delivery against fungal infections.

\section{ACKNOWLEDGEMENT}

The authors are thankful to Ranbaxy Laboratory, New Delhi for providing sulconazole nitrate as gift sample and N.G.S.M institute of pharmaceutical sciences, Nitte (Deemed to be University), Mangaluru for providing the necessary facilities to carry out this work.

\section{CONFLICT OF INTEREST}

The authors declare that there is conflict of interest.

\section{REFERENCES}

1. Kikwai L, Babu RJ, Prado R, Kolot A.In vitro and In vivo evaluation of topical formulation of spantide 2. AAPS Pharm. Sci. Tech. 2005;6(4):34-39.

2. Kumar $R$, Katare $O P$, Lecithin organogels as a potential phospholipid structured for topical drug delivery, a review. AAPS Pharm. Sci. Tech. 2005;06(02):56-61.

3. Chein YW. Transdermal therapeutic systems. In: Robinson JR, Lee VHL, eds.Text book of Controlled drug delivery: fundamentals and application $\left(2^{\text {nd }} e d\right)$. New York;Marcel Dekker Inc; 1987:524-549.

4. Chen $\mathrm{H}$. Microemulsion based hydrogel formulation of ibuprofen for topical delivery. Int. J. Pharm. 2006 Feb;315:52-58. 
5. Benjamin EJ, Lee Maryann. Stabilization of sulconazole nitrate in topical powder formulation. Int J Pharma. 1983;14:209-211.

6. Badawi AA, Nour SA, Sakran WS, El-Mancy SMS. Preparation and evaluation of microemulsion systems containing salicylic acid. AAPS Pharm. Sci. Tech. 2009 Dec;10(4):1081-1084.

7. Brime B, Moreno M. Amphotericin B in oil in water Lecithin-based microemulsions: Formulation and toxicity evaluation. J. Pharma. Sci. 2002;91(4):1178-1185.

8. Karasulu YH, Karabulut B, Goker E. Controlled release of Methotrexate from w/o microemulsion and its in vitro antitumor activity. Informa healthcare 2006:225-233.

9. Bajpai M, Sharma PK, Mittal A. A study of oleic acid base for the tropical delivery of dexamethasone microemulsion formulations. Asian J. Pharm 2009 July-Sep; 208214.

10. Dhamankar AK, Manwar JV, Kumbhar DD. The novel formulation design of o/w microemulsion of ketoprofen for improving transdermal absorption. Int. J. Pharm. 2009 Oct-Dec;1(4):1449-1457.

11. Chen $\mathrm{H}$. Microemulsion based hydrogel formulation of ibuprofen for topical delivery. Int. J. Pharm. 2006 Feb;315:52-58

12. Basha NB, Prakasam K, Goli D. Formulation and evaluation of gel containing fluconazole-antifungal agent. Int J Drug Dev\& Res. 2011 Oct;3(4):109-28

13. Kumar A, Kushwaha V, Sharma PK. Pharmaceutical microemulsion: Formulation, characterization and drug deliveries across skin. Int J Drug Dev Res 2014;6(1):1-21. 